University of Wollongong

Research Online

Faculty of Health and Behavioural Sciences -

Papers (Archive)

Faculty of Science, Medicine and Health

$1-1-2012$

\title{
Beneficial effects of a high-protein, low-glycemic-load hypocaloric diet in overweight and obese women with polycystic ovary syndrome: a randomized controlled intervention study
}

\author{
Homeira Hamayeli Mehrabani \\ National Nutrition and Food Technology Research Institute \\ Saghar Salehpour \\ Shahid Beheshti University of Medical Sciences \\ Zohreh Amiri \\ National Nutrition and Food Technology Research Institute \\ Sara Jalali Farahani \\ Shahid Beheshti University of Medical Sciences \\ Barbara J. Meyer \\ University of Wollongong, bmeyer@uow.edu.au
}

See next page for additional authors

Follow this and additional works at: https://ro.uow.edu.au/hbspapers

Part of the Arts and Humanities Commons, Life Sciences Commons, Medicine and Health Sciences Commons, and the Social and Behavioral Sciences Commons

\section{Recommended Citation}

Mehrabani, Homeira Hamayeli; Salehpour, Saghar; Amiri, Zohreh; Farahani, Sara Jalali; Meyer, Barbara J.; and Tahbaz, Farideh: Beneficial effects of a high-protein, low-glycemic-load hypocaloric diet in overweight and obese women with polycystic ovary syndrome: a randomized controlled intervention study 2012. https://ro.uow.edu.au/hbspapers/3199 


\title{
Beneficial effects of a high-protein, low-glycemic-load hypocaloric diet in overweight and obese women with polycystic ovary syndrome: a randomized controlled intervention study
}

\begin{abstract}
OBJECTIVE: The recommended composition of a hypocaloric diet for obese women with polycystic ovary syndrome (PCOS) is unclear. The aim of this study was to investigate the effects of a high-protein, lowglycemic-load diet compared with a conventional hypocaloric diet on reproductive hormones, inflammatory markers, lipids, glucose, and insulin levels in obese women with PCOS. METHODS: A total of 60 overweight and obese women with PCOS who did not use insulin-sensitizing agents were recruited and randomly assigned to 1 of the 2 hypocaloric diet groups for a single-blind clinical trial. The groups included a conventional hypocaloric diet (CHCD) (15\% of daily energy from protein) and a modified hypocaloric diet (MHCD) with a high-protein, low-glycemic load ( $30 \%$ of daily energy from protein plus low-glycemic-load foods selected from a list) that was prescribed via counseling visits weekly during 12 weeks of study. Anthropometric assessments and biochemical measurements including reproductive hormones, inflammatory factors, lipids, glucose, and insulin were performed on fasting blood samples at baseline and after 12 weeks of dietary intervention. RESULTS: Weight loss was significant and similar in the 2 groups. Mean of testosterone in the MHCD and CHCD groups decreased from $1.78 \pm 0.32$ to $1.31 \pm$ $0.26 \mathrm{ng} / \mathrm{ml}$ and from $1.51 \pm 0.12$ to $1.15 \pm 0.11 \mathrm{ng} / \mathrm{ml}$, respectively $(p<0.001)$. Follicle sensitizing hormone (FSH), luteinizing hormone (LH), and blood lipids concentrations were not changed except lowdensity lipoprotein cholesterol (LDL-C) was reduced by $24.5 \% \pm 12.3 \%$ ( $p<0.001$ for both) after 12 weeks of intervention. MHCD resulted in a significant reduction in insulin level, homeostatic model assessment for insulin resistance (HOMA), and high-sensitivity $C$ - reactive protein (hsCRP) concentration $(p<0.001)$. CONCLUSIONS: In this study both hypocaloric diets significantly led to reduced body weight and androgen levels in these two groups of women with PCOS. The combination of high-protein and low-glycemic-load foods in a modified diet caused a significant increase in insulin sensitivity and a decrease in hsCRP level when compared with a conventional diet
\end{abstract}

Disciplines

Arts and Humanities | Life Sciences | Medicine and Health Sciences | Social and Behavioral Sciences

Publication Details

Mehrabani, H. Hamayeli., Salehpour, S., Amiri, Z., Farahani, S. Jalali., Meyer, B. J. \& Tahbaz, F. (2012). Beneficial effects of a high-protein, low-glycemic-load hypocaloric diet in overweight and obese women with polycystic ovary syndrome: a randomized controlled intervention study. Journal of the American College of Nutrition, 31 (2), 117-125.

\section{Authors}

Homeira Hamayeli Mehrabani, Saghar Salehpour, Zohreh Amiri, Sara Jalali Farahani, Barbara J. Meyer, and Farideh Tahbaz 


\section{Beneficial effects of a High Protein Low Glycemic Load Hypocaloric Diet in overweight and obese women with polycystic ovary syndrome: A randomized controlled intervention study}

${ }^{1}$ Homeira Hamayeli Mehrabani PhD, ${ }^{2}$ Saghar Salehpour MD, ${ }^{1}$ Zohreh Amiri PhD, ${ }^{2}$ Sara Jalali Farahani BS, ${ }^{3}$ Barbara J. Meyer PhD, ${ }^{1}$ Farideh Tahbaz* $\mathrm{PhD}$

Names and affiliations of the authors:

${ }^{1}$ Homeira Hamayeli Mehrabani, $\mathrm{PhD}$

Faculty of Nutrition Sciences and Food Technology, National Nutrition and Food Technology Research Institute, Shahid Beheshti University of Medical Sciences, PO Box 19395/4741

Tehran/Iran

${ }^{2}$ Saghar Salehpour, MD

Associate Professor, Infertility fellow, Faculty of Medicine, Shahid Beheshti University of Medical Sciences, PO Box : 19395/1389

Tehran/Iran

${ }^{1}$ Zohreh Amiri, PhD

Assistant Professor, Statistician, Faculty of Nutrition Sciences and Food Technology, National Nutrition and Food Technology Research Institute, Shahid Beheshti University of Medical Sciences, PO Box 19395/4741

Tehran/Iran

${ }^{2}$ Sara Jalali Farahani, BS in Nutrition

Endocrine Research Center, nutrition, Tehran, Iran, Islamic Republic of, Shahid Beheshti University of Medical Sciences,

PO Box : 19395/1389

Tehran/Iran

${ }^{3}$ Barbara J. Meyer, PhD

Associate Professor, School of Health Sciences, University of Wollongong, Northfields Ave, Wollongong, NSW, 2500, Australia

${ }^{1}$ Farideh Tahbaz, PhD 
Associate Professor, Faculty of Nutrition Sciences and Food Technology, National Nutrition and Food Technology Research Institute, Shahid Beheshti University of Medical Sciences, PO Box 19395/4741

Tehran/Iran

\section{*Corresponding author:}

Farideh Tahbaz, PhD

Department of Clinical Nutrition and Dietetics, Faculty of Nutrition Sciences and Food Technology, National Nutrition and Food Technology Research Institute, Shahid Beheshti University of Medical Sciences, PO Box 19395/4741,

Tehran/Iran.

Email: farideh.tahbaz@gmail.com

Tel: +982122077424

Fax: +982122360660

Source of funding: National Nutrition and Food Technology Research Institute, Shahid Beheshti University of Medical Sciences, Tehran, Iran

Conflict of interest: There are no conflicts of interest between the authors and the source of funding. All coauthors accept responsibility for content of the manuscript.

Running Title: Effects of high protein low GL diet on PCOS women 


\title{
Beneficial effects of a High Protein Low Glycemic Load Hypocaloric Diet in overweight and obese women with polycystic ovary syndrome: A randomized controlled intervention study
}

\begin{abstract}
Objective: The recommended composition of a hypocaloric diet for obese women with polycystic ovary syndrome (PCOS) is unclear. The aim of this study was to investigate the effects of a high protein- low glycaemic load diet compared to a conventional hypocaloric diet on reproductive hormones, inflammatory markers, lipids, glucose and insulin levels in obese women with PCOS.

Methods: Sixty overweight and obese women with PCOS who did not use insulin sensitizing agents were recruited and randomly assigned in one of the two hypocaloric diet groups for a single, blinded, clinical trial. The groups included; conventional hypocaloric diet (CHCD) (15\% of daily energy from protein) and modified hypocaloric diet (MHCD) with high protein-low glycaemic load (30\% of daily energy from protein plus low glycaemic load foods selected from a list) which was prescribed via counseling visits weekly during 12 weeks of study. Anthropometric assessments and biochemical measurements including reproductive hormones, inflammatory factors, lipids, glucose and insulin were performed on fasting blood samples at baseline and after 12 weeks of dietary intervention.

Results: Weight loss was significant and similar in two groups. Mean of testosterone in MHCD and CHCD group decreased from $1.78 \pm 0.32$ to $1.31 \pm 0.26 \mathrm{ng} / \mathrm{ml}$ and from $1.51 \pm 0.12$ to $1.15 \pm 0.11 \mathrm{ng} / \mathrm{ml}$, respectively $(\mathrm{p}<0.001)$. Follicle sensitizing hormone (FSH), Luteinizing hormone (LH), blood lipids concentrations were not changed except -Low density lipoprotein cholesterol (LDL-C) was reduced by $24.5 \% \pm 12.3 \quad(\mathrm{p}<0.001$ for both) after 12 weeks of intervention. MHCD resulted in a significant reduction in insulin level, homeostatic model assessment for Insulin resistance (HOMA) and high sensitivity $\mathrm{C}$ - reactive protein (hsCRP) concentration $(\mathrm{p}<0.001)$.

Conclusions: In this study both hypocaloric diets significantly lead to reduced body weight and androgen levels in these two groups of women with PCOS. The combination of high protein and low glycemic load foods in modified diet caused a significant increase in insulin sensitivity and a decrease in hsCRP level when compared to the conventional diet.
\end{abstract}

Keywords: PCOS, hypocaloric diet, hormones, inflammatory factors, blood lipids, glycaemic load, dietary protein 


\section{Introduction:}

Polycystic ovary syndrome (PCOS) was first described more than 70 years ago [1]. It is a hormonal imbalance which is generally thought to affect approximately 1 out of 16 women [2]. The majority of these women are obese [2]. Obesity can exacerbate the metabolic and reproductive abnormalities that are associated with the disorder, mostly by increasing insulin resistance and hyperinsulinemia; so weight loss accompanied by an increase in insulin sensitivity was highly recommended for this population group [3]. The ideal hypocaloric diet should decrease body mass, improve cardiovascular risk factors, and decrease fat tissue while sparing lean tissue.

Low carbohydrate diets were traditionally thought to aid weight loss and improve metabolic and reproductive dysfunction [4]. An increasing interest in a high protein/low carbohydrate diet has been observed [5]. Stamets et al. compared the short- term effect of a high protein diet (30\% protein and $40 \%$ carbohydrate) or a high carbohydrate diet (15\% protein and $55 \%$ carbohydrate) on weight loss in obese PCOS [6]. Both groups lost approximately 3.6\% (high protein group) and $4.2 \%$ (high carbohydrate group) of body weight, but there were no significant differences between the diets [6]. Douglas et al., used eucaloric diets either with mono-unsaturated fatty acids (17\% of energy) or low carbohydrate ( $43 \%$ of energy) relative to standard diet $(56 \%$ carbohydrate, $31 \%$ fat and $16 \%$ protein) over 16 days, which resulted in lower fasting insulin and acute insulin response to glucose in low carbohydrate diet compared to the standard and monounsaturated fatty acids diets, respectively [7]. Moran et al., studied high protein (30\% of total calories) versus low protein (15\% of total calories) plus exercise for 12 weeks followed by a 4 week weight maintenance in obese subjects with PCOS. Both groups lost approximately $7.7 \mathrm{~kg}$ of weight and there was no effect of diet composition [8]. 
Although high protein diet did not make any significant independent difference in comparison to other weight loss diets, it seems that protein intake induces satiety and enhances the feeling of well- being and also self -esteem for obese PCOS who have tried to lose weight [9]. The concept of reducing glycaemic index (GI) and glycaemic load (GL) in the treatment of obese PCOS has received considerable interest. GI refers a system for classifying the glycaemic response of carbohydrate and measures the relative area under the postprandial glucose curve of $50 \mathrm{~g}$ of digestible carbohydrates compared with $50 \mathrm{~g}$ of a standard food, either glucose or white bread. The GL which developed later is calculated by multiplying the GI by the amount of carbohydrates in each food [10].

In 2008 Herriot et al performed a retrospective audit on a group of women with PCOS who had been advised to follow a low calorie- low GL diet over a 2 year period. They found that this type of diet with $40-45 \%$ of daily energy intake from carbohydrate, $30 \%$ from protein and about $10 \%$ from saturated fat together with insulin sensitizing medications caused weight loss and better weight maintenance in overweight and obese patients with PCOS [11]. However the impacts of the prescribed diets like many similar studies were limited to subjects' self reporting and there was a lack of thorough investigation about the final biochemical and anthropometric results.

Thus the aim of this controlled intervention study was to investigate and compare the effects of two kinds of diets including Conventional Hypocaloric Diet (CHCD) and Modified Hypocaloric Diet (MHCD) with low GL and high protein content on reproductive hormones, lipid profile, inflammatory factors, glucose and insulin levels in overweight and obese women with PCOS who had not started taking any medications or changed their exercise habits since the diagnosis of their disease.

\section{Materials and methods:}


A total of 60 women were recruited through newspaper advertisement, brochures and referral of gynecologist in an out- patient nutrition clinic visits in Taleghani infertility research center of Tehran, Iran.

Subjects were included if they were aged between 20 and 40 years, had a body mass index (BMI) greater than 25 and less than $38 \mathrm{~kg} / \mathrm{m}^{2}$, and no history of using insulin sensitizing agent such as metformin or oral contraceptives. Inclusion criteria were diagnosis of PCOS by menstrual irregularity (cycle length $<21$ days or $>35$ days), hirsutism and biochemical hyperandrogenism. Volunteers were excluded if they were smokers, exercised heavily, and/or had any history of cardiovascular, renal, gastrointestinal disorders, liver or metabolic diseases. Women diagnosed with hyperprolactinemia, thyroid abnormalities, and/or non classic adrenal hyperplasia were also excluded from this study.

The study protocol was approved by the ethics committee of the Institute of Nutrition Sciences and Food Technology of Iran and all participants accepted the conditions of the study and gave their informed written consent.

\section{Study design}

It was calculated that 23 subjects per group would provide $80 \%$ power to detect a difference of $7.7 \mathrm{nmol} / \mathrm{L}$ in the sex hormone binding globulin (SHBG) levels in serum. Therefore 30 subjects were recruited for each group to cover the assumed withdrawal. Subjects were stratified to ensure equality of distribution in two groups for known confounding factors like age and BMI, and then randomized by an independent observer. Investigator was not blinded as to the kind of dietary intervention, but subjects were.

Dietary intervention for two groups; were the Conventional Hypocaloric Diet (CHCD) and the Modified Hypocaloric Diet (MHCD) each for 12 weeks. A dietitian initially instructed the 
subjects on quantification and recording of their daily food intakes. They were followed weekly and asked to prepare dietary records for 3 days every month during the intervention (weeks 0,4 , 8 and 12) to enhance compliance and to enable the dietitian monitoring the prescribed diet. Subjects were requested not to change their regular physical activities during the study period. In order to check that they were asked to record all their daily physical activity and resting hours at the beginning and during the intervention.

\section{Description of the diets}

For both groups energy content of hypocaloric diet was calculated on the basis of subject's BMI of $21-22 \mathrm{~kg} / \mathrm{m}^{2}$ and a deficit of 500 to $1000 \mathrm{kcal}(2090-4180 \mathrm{~kJ})$ per day. CHCD consisted of $55 \%$ of energy from carbohydrate, $15 \%$ of energy from protein and 30\% energy from fat. MHCD included $40 \%$ of energy from low and medium glycemic load carbohydrates, $30 \%$ of energy from protein and $30 \%$ of energy from fat, with limitation of high glycemic foods. The MHCD group refrained from consuming high glycemic load foods (GL $\geq 20)$. These foods in addition to the GL of the other foods were provided to them in a list [12]. In order to assist the subjects in meal planning some menu including poultry, fish, legumes, egg white, fruits and non starchy vegetables which were similar to their regular dietary habits were suggested by the researcher. In these meals the contents of macronutrients were close to the goals of the intervention. Furthermore the subjects were instructed about exchanging food items and to insure about their diet compliance they were followed by weekly counseling visits.

Both groups of patients were precisely instructed about the foods that were or were not permitted to consume. Eating habits of them were assessed using a food frequency questionnaire [13].

\section{Anthropometric assessments}


All the anthropometric measurements were assessed in the fasting state. Body composition including total body fat and lean body mass were measured at the beginning and the end of the intervention using bioelectrical impedance (BioStat Co.,1500, UK). Skinfold thickness was measured at suprailiac area using skinfold caliper (Vogel, Germany) in order to determine abdominal fatness. Waist circumference was measured at area demarcated by umbilicus and at the widest place over the buttocks for hip circumference, by a non- stretchable tape. Subjects wore light clothes without shoes when they were weighed (Seca weighing scale, Model 709, Seca GmbH \& co. kg. Germany) and their height were measured without shoes.

\section{Biochemical measurements}

After an overnight fast, $10 \mathrm{ml}$ blood samples were taken. The test was performed either at random in the amenorrheic women or 2 to 5 days after menstruation. Follicle stimulating hormone (FSH), luteinizing hormone (LH), sex hormone binding globulin (SHBG) were measured by IEMA (Immuno Enzymmometric Assay) method and, estradiol, total testosterone, dehydroepiandrosterone sulfate (DHEAS) and androstenedione were determined by Enzyme Immuno Assay (EIA ) using a commercially available kit in serum (Diagnostics Biochem Canada Inc., Ontario, Canada)[14]. The coefficients of variance (CVs) for these assays were as follows: $2.1 \%$ for FSH, $4.1 \%$ for $\mathrm{LH}, 3.1 \%$ for SHBG, $6.2 \%$ for DHEAS, $5 \%$ for total testosterone, and 5.2\% for androstenedione. Fasting insulin and adiponectin were measured using ELISA( Enzyme-Linked Immunosorbent Assay) (Mercodia, Uppsala, Sweden) and CVs were $7 \%$ and $5.1 \%$ respectively. Serum glucose, triglyceride, total cholesterol and high density lipoprotein cholesterol (HDL-C) were measured by a colorimetric enzymatic assay and the CV was lower than $2.5 \%$. Friedewald equation used for low density lipoprotein cholesterol (LDL-C) estimation [15]. Interleukin-6 (IL-6), Tumor Necrosis Factor-Alpha (TNF $\alpha$ ) were measured 
using ELISA (Diaclone, Besancon, France) and their CVs were 5.8\% and 7.9\% respectively, high sensitivity C- reactive protein (hsCRP) was measured by ELISA (DBC, Ontario, Canada) with CV 9.8\%. HOMA (Homeostatic Model Assessment for Insulin Resistance) was used as a surrogate measure of insulin sensitivity and was calculated as [fasting serum insulin $(\mathrm{mU} / \mathrm{L}) \times$ fasting serum glucose (mmol/L)/ 22.5] [16]. Free androgen index (FAI) was calculated using the formula $\mathrm{FAI}=\mathrm{T} / \mathrm{SHBG} \times 100[17]$.

\section{Statistical analyses}

Data were analysed using the statistics package for social sciences 15 (SPSS, Chicago, IL, USA). Baseline parametric and non- parametric characteristics of subjects in two groups and also the differences between two groups (week 12 minus baseline) were compared by t-test and MannWhitney test. Paired- t- test were performed to evaluate the possible differences between baseline and week 12 for each group, separately. P value of $<0.05$ was considered as significant.

The results were analysed in two ways. Firstly a paired t-test was used to compare baseline and week 12 data. Secondly, the data of week 12 minus baseline were compared between the 2 groups.

\section{Results}

In total 49 out of 60 subjects completed the study, 26 in CHCD and 23 in MHCD. Three had difficulty with blood sample draws, 2 could not tolerate any diet, 1 changed her mind and wanted to begin IVF processing (In Vitro Fertilization), 2 had family reasons, 1 moved out of the city without any forwarding address, and 2 did not provide any reason.

No significant differences existed in subjects' characteristics at baseline for two treatment groups, except for hip circumference (table 1). Both the MHCD and CHCD diets resulted in 
significant weight loss $(p<0.05)$. None of the subjects had weight gain during the study. The range in weight loss was -2 to $-10 \mathrm{~kg}$ for $\mathrm{MHCD}$ and -1 to $-9 \mathrm{~kg}$ weight loss for the $\mathrm{CHCD}$ group. It was estimated $4.1 \% \pm 0.58 \%$ and $3.3 \% \pm 0.62 \%$ weight loss for MHCD and $\mathrm{CHCD}$, respectively in regards to initial weight, without any significant differences. Subjects in both groups did not change their physical activity during the study. Although there was not any significant changes in total body fat and lean body mass based on BIA after 12 weeks of manipulation of weight reduction diet, but in both groups fat mass was reduced at the end of the intervention (Fig.1).

The reduction of waist circumference in MHCD was significantly more than CHCD $(p<0.05)$. Dietary intakes at baseline and after intervention are shown in table 2 . There were no significant differences in dietary intakes of two groups (zero time). During intervention, daily energy intakes decreased with no significant difference between the two. As expected, protein as percentage of energy intake was higher and carbohydrate was lower in MHCD, and these differed significantly in comparison to $\mathrm{CHCD}$. Contribution of fat in total energy intake decreased in both groups. Carbohydrate and total fat intakes were decreased for MHCD and replaced by protein. Fiber intake increased by approximately $20 \%$ in MHCD group $(p<0.05)$ but no difference was observed in fiber intake in the CHCD group. There was not any significant difference between the changes of fiber intake in two groups at week $12(0.3 \pm 1.4 \mathrm{~g}$ for $\mathrm{CHCD}$ vs. $2.8 \pm 1.4 \mathrm{~g}$ for MHCD).

There was no effect of energy reduction or diet composition on FSH, LH and blood lipids except for LDL-cholesterol which decreased significantly in both groups (table 3). Reduction of LDLcholesterol levels were $23.7 \% \pm 13.8 \%$ for $\mathrm{CHCD}$ and $25.5 \% \pm 10.5 \%$ for MHCD without any significant differences between the two groups. Mean \pm SE of fasting glucose for CHCD vs. 
MHCD, respectively at baseline $96.0 \pm 1.5$ vs. $97.4 \pm 1.4 \mathrm{mg} / \mathrm{dl}(p=0.49)$ and week 12 was $98.3 \pm 1.7$ vs. $98.5 \pm 2.5 \mathrm{mg} / \mathrm{dl}(p=0.95)$.

SHBG increased and testosterone, DHEAS decreased in both groups (Table3). There were no significant differences between two groups in regard to the changes of reproductive hormones (Table 3).

In addition there were substantial decreases in fasting insulin level and HOMA after 12 weeks for both kinds of interventional diets; $25.3 \%$ and $23.2 \%$ for MHCD $(p<0.001)$ vs. $5.9 \%$ and $4.2 \%$ for $\mathrm{CHCD}(p<0.001)$. However this only remained significant after adjusting for BMI changes in MHCD and found to be related directly to the type of diet. TNF $\alpha$ decreased and adiponectin increased significantly in both groups (table 4), IL-6 did not change and hsCRP decreased in MHCD, which was significant after adjusting for baseline hsCRP.

\section{Discussion:}

This study demonstrates that both kinds of hypocaloric diets with no medications and instructed physical exercise resulted in a significant weight loss, lowering of LDL-cholesterol, reduction of androgens and an increase in SHBG, but consumption of the MHCD a hypocaloric diet with low GL carbohydrates and high protein content induced concomitant reductions of insulin and HOMA and therefore would be viewed as more favorable than the CHCD.

The attrition rate in this study was about $20 \%$ which was much lower than this rate in a recent clinical study investigating the effects of low GI diet on women with PCOS [18]. Perhaps prescribing the diets according to the patients' regular dietary habits and without any medications might be a factor in this higher adherence of patients to their diets in this study. This is shown in these subjects who achieved the macronutrient goals which were set for them through their diets. Effects of hypocaloric diets on weight loss 
Even though the benefit of weight loss for women with PCOS is clear but the ideal diet composition for their management is not known. There is a paucity in the study of reduced GL diets for obese PCOS women. In a retrospective study of normal and obese PCOS by Herriot et al., weight and fasting glucose were significantly reduced without changes in insulin concentrations for 13 patients by a low GL load diet low GL foods, reduced carbohydrate of approximately $40-45 \%$ energy, $30 \%$ protein, reduced saturated fat of approximately $10 \%$ and no snacking. The audit indicated that a low GL diet may contribute to an improvement in symptom relief in patients with PCOS such as carbohydrate craving and hypoglycemia [11].

In this study weight loss was similar in the two groups and MHCD did not have any superior effect on weight loss compared to CHCD. But MHCD results in a higher decrease of waist and hip circumferences that might be due to the lower GL of carbohydrates or higher protein intakes; although at baseline the hip circumferences in MHCD had a significant difference with CHCD and could explain significant differences at week 12 between MHCD and CHCD. In the other studies the weight reduction was not different for high protein $(30 \%)$ and low protein (15\%) diets $[8,9]$. In the study performed by Moran et al [8] weight loss on high protein and low protein diets for the same duration were much higher than this current study. It can be assumed that regular class and exercise in their study were the key contributing factors for similar and higher degrees of weight loss in both groups.

Few studies have addressed the effects of varying diet composition on the obese PCOS population; hypocaloric diet supplemented with protein reduced fat mass and body weight more than diet supplemented with simple sugars [19]. It seems that the subjects receiving higher protein may have more successful weight loss because of satiating effect of diet $[9,20]$, but there were no significant differences in hormonal changes attributed to the composition of diet [6]. 


\section{Effects of hypocaloric diets on reproductive hormones and insulin levels}

The improvement of the abnormal reproductive hormones in this current study were consistent with some studies which had observed the effect of weight loss in improving abnormal reproductive and metabolic variables in women with PCOS [6, 8, 18 and 21]. It is currently unclear why reproductive function is restored with energy restriction or weight loss, how long it would take for the hormonal changes to translate into improvements in reproductive functions, and what the key triggering hormonal factors are [21].

In this study it is hypothesized that a hypocaloric diet with increased protein content and reduced GL would decrease insulin concentration and this in turn, results in lowering androgen concentrations. The levels of total testosterone and DHEAS decreased similarly in both groups after weight loss. In spite of significant decreases of insulin and HOMA in the MHCD group, there was no effect of reduced insulin on androgen levels. It seems that the changes in insulin concentration in this study were not enough to result in the reduction of androgens. Perhaps this was related to insulin resistance, or lower insulin sensitivity in some of the studied subjects, but these were not measured directly.

Similar to the current study, Moran et al. found no significant effect of diet composition on testosterone or SHBG levels and showed that endocrine improvements caused maximal changes in insulin sensitivity, suggesting a relationship between the two [8]. Decreasing in the level of fasting insulin, increasing in SHBG level, and decreasing in testosterone were related to energy restriction diets. It is possible that the family of Insulin like Growth Factors (IGFs) and their binding proteins may be involved [22], particularly IGF- binding protein-I increases during short term energy restriction mediated by decreased insulin levels. Increased IGF- binding protein-I 
decreases free IGF-I, which down regulates androgen synthesis through the cytochrome P450c17 system [23].

Free testosterone concentrations are reported to decline significantly following weight loss and decrease of insulin concentration [24]. Previously Pasquali et al. showed that moderate weight loss resulted in a reduction in insulin levels, but did not alter androgen and DHEAS levels [25]. There were no effects of weight loss or type of diet seen on LH and FSH. There were no changes of these hormones seen in other studies $[8,26]$. Guzick et al. observed no effect of weight loss in obese hyperandrogenic women and suggested that weight loss had no hypothalamic effect for secretion of LH. It is believed that LH concentration is not related to insulin concentration [27]. Moreover this current study showed a significant effect of MHCD on insulin concentration and HOMA when compared with CHCD. This suggests that the lower GL in the MHCD group in combination with higher protein, improved insulin resistance, as low GL has been shown to improve insulin resistance $[28,29]$. In contrast, the Moran et al. study showed improvements in insulin concentration and HOMA in both energy restricted diets (high carbohydrate/low protein and high protein/low carbohydrate) [8]; however, in their study, both groups increased their exercise levels and it has been shown that exercise improves insulin resistance in PCOS women [30]. These two studies taken together one could speculate that to improve insulin levels and HOMA in women with PCOS, the women either go on an energy restricted diet (irrespective of composition) so long as they also increase their exercise [8] or the women go on a MHCD diet without exercise as shown in this study. A limitation of the Moran et al study was the lack of an exercise only arm [8], which would have definitively shown that exercise was responsible for the improvements in insulin levels and HOMA.

\section{Effects on cardiovascular risk factors:}


It has been proposed that excess fat accumulation alters the pattern of adipokines secretion causing a low- grade inflammatory state that induces insulin resistance and endothelial dysfunction leading to diabetes and cardiovascular disease [31] and conversely fat mass loss or weight reduction induce decreasing in cardiovascular risk factors. Some studies showed the relation between change of insulin resistance and decreasing in these risk factors [32, 33] but it has been assumed that the levels of blood lipids and inflammatory factor at baseline are important for observing a significant reduction.

Noakes et al. reported decrease of serum triacylglycerol after weight loss in obese women with high baseline triglycerides [34]. Moran et al also showed reductions in plasma triacylglycerol after weight loss (due to energy restricted diets and increased exercise) on women with PCOS [8]. In the current study there were no significant differences in plasma triacylglycerol but this could be explained by the baseline plasma triacylglycerol levels being approximately $20 \%$ lower than the baseline plasma triacylglycerols in the study by Moran et al [8]. However, in the current study LDL-C decreased in both groups, and this could be due to its higher levels at the beginning of the study. Previously dietary GL and carbohydrate has been associated with a number of cardiovascular risk factors, reduced HDL-C and elevated triacylglycerol concentrations and also hsCRP level $[35,36]$.

There are limited trials for inflammatory factors in obese PCOS women after weight loss by hypocaloric diet. CRP was reduced significantly after consumption of an energy restricted diet for 8 weeks [21]. But in comparison between women with and without PCOS, there were a significant interaction between PCOS status and CRP such that CRP did not decrease with about $4 \%$ weight loss in PCOS women and TNF $\alpha$ increased after weight loss which was surprising because usually inflammatory factors decrease after weight loss. Esposito et al. reported 
reduction of inflammatory factors by using a hypocaloric Mediterranean style diet and regular physical activity in obese women [37]. In the current study TNF $\alpha$ decreased for both groups and IL-6 did not change and hsCRP decreased only in MHCD. In addition decrease in waist circumference was higher in MHCD than in CHCD, so reduction of CRP maybe related to this variable. Linear regression analysis revealed hsCRP baseline and waist difference parameter and individual variability has been suggested to have an important influence on the results [38]. The decrease in hsCRP concentration after weight reduction does not appear to be mediated by decrease in circulating IL-6 or adiponectin concentrations [39]. Adiponectin increased in both group after weight loss which had observed in the other studies [37] but it seems that the change in adiponectin level after weight loss did not happen regularly in obese PCOS women [38, 40]. It has been suggested to study the ratio of high molecular weight adiponectin to low molecular weight adiponectin because of the varying appearance of the two adiponectin oligomers on fat and glucose metabolism [41].

In conclusion the current study demonstrated that two kinds of low calorie diets in women with PCOS who had not started taking any medications and without any prescribed exercise have significant effects on testosterone, androstenedione, dehydroepiandrosterone, SHBG, FAI, LDL$\mathrm{C}$, adiponectin, $\mathrm{TNF} \alpha$ and to our knowledge, this is the first interventional study to suggest that high protein plus low GL hypocaloric diet without exercise has additional effects on lowering insulin level and HOMA.

However the limitation of this study was that it was a self- reported study in which the researchers relied on the study participants for following their prescribed diets, and hence controlled-feeding programs for future studies is recommended. Recommendations for future studies include considering abdominal fat in addition to BMI as an inclusion criterion, since this 
way the subjects would be metabolically much more similar. It is also recommended to follow up patients for another 6 months to see the weight maintenance and the consequences of weight reduction on the reproduction of these groups of women.

\section{Conclusion}

Both hypocaloric diets without using any medications and instructed exercise were shown to significantly reduce body weight and androgens levels in two groups of women with PCOS. In addition a high protein-low GL diet caused a significant increase in insulin sensitivity and a decrease in hsCRP level when compared to conventional diet. However a study to differentiate the effects of protein content and glycemic load in a hypocaloric diet for women with PCOS is needed. 


\section{References:}

1- Stein IF, Leventhal ML: Amenorrhoea associated with bilateral polycystic ovaries. Am J Obstet Gynecol 29: 181-91, 1935.

2- Duniaf A, Thomas A: Current concepts in the polycystic ovary syndrome. Annu Rev Med 52, 401-19, 2001.

3- Wild RA, Painter PC, Coulson PB, Carruth KB, Ranney GB: Lipoprotein lipid concentrations and cardiovascular risk in women with polycystic ovary syndrome. J Clin Endocrinol Metab 61: 946-51, 1985.

4- Raatz SK, Torkelson CJ, Redmon JB, Reck KP, Kwong CA, Swanson JE, Liu C, Thomas W, Bantle JP: Reduced glycemic index and glycemic load diets don't increase the effects of energy restriction on weight loss and insulin sensitivity in obese men and women. J Nutr 135: 2387-91, 2005.

5- Meckling KA, Sherfey R: A randomized trial of hypocaloric high - protein diet, with and without exercise, on weight loss, fitness, and markers of the metabolic syndrome in overweight and obese women. Appl Physiol Nutr Metab 32:743-52, 2007.

6- Stamets K, Taylor DS, Kunselman A, Demers LM, Pelkman CL, Legro RS: A randomized trial of the effects of two types of short- term hypocaloric diets on weight loss in women with polycystic ovary syndrome. Fertil Steril 81: 630-7, 2004.

7- Douglas CC, Gower BA, Darnell BE, Ovalle F, Oster RA, Azziz R: Role of diet in the treatment of polycystic ovary syndrome. Fertil Steril 85: 679-88, 2006.

8- Moran LJ, Noakes M, Clifton PM, Tomlinson CL, Galletly C, Norman RJ: Dietary composition in restoring reproductive and metabolic physiology in overweight women with polycystic ovary syndrome. J Clin Endocrinol Metab 88: 812-9, 2003. 
9- Galletly C, Moran L, Noakes M, Clifton P, Tomlinson L, Norman R: Psychological benefits of a high- protein, low- carbohydrate diet in obese women with polycystic ovary syndrome- a pilot study. Appetite 49: 590-93, 2007.

10- Gallagher ML: The nutrients and their metabolism. In: Escott-Stump S, Mahan LK (eds): “Krause's food and nutrition therapy,” 12th ed. Philadelphia: Saunders; pp 50, 2008.

11- Herriot AM, Whitcroft S, Jeanes Y: An retrospective audit of patients with polycystic ovary syndrome: the effects of a reduced glycaemic load diet. J Hum Nutr Diet 21: 337-45, 2008.

12- Foster-Powell K, Holt SH, Brand-Miller JC: International table of glycemic index and glycemic load values: 2002. Am J Clin Nutr 76: 5-56, 2002.

13- Mirmiran P, Esfahani FH, Mehrabi Y, Hedayati M, Azizi F: Reliability and relative validity of an FFQ for nutrients in the Tehran lipid and glucose study. Public Health Nutr 13: 654-62, 2010.

14- Edwards R: “Immunoassays” New York: John Eiley \& Sons, 1996.

15- Friedewald WT, Levy RI, Fredrickson DS: Estimation of the concentration of low-density lipoprotein cholesterol in plasma, without use of the preparative ultracentrifuge. Clin Chem 18: 499-502, 1972.

16- Matthews DR, Hosker JP, Rudenski AS, Naylor BA, Treacher DF, Turner RC: Homeostasis model assessment: insulin resistance and beta cell function from fasting plasma glucose and insulin concentrations in man. Diabetologia 28: 412-9, 1985.

17- Vermeulen A, Verdonck L, Kaufman JM: A critical evaluation of simple methods for the estimation of free testosterone in serum. J Clin Endocrinol Metab 84: 3666-72, 1999. 
18-Marsh KA, Steinbeck KS, Atkinson FS, Petocz P, Brand-Miller JC: Effect of a low glycemic index compared with a conventional healthy diet on polycystic ovary syndrome. Am J Clin Nutr 92: 83-92, 2010.

19- Kasim- Karakas SE, Almario RU, Cunningham W: Effects of protein versus simple sugar intake on weight loss in polycystic ovary syndrome. Fertil Steril 92: 262-70, 2009.

20- Pasquali R, Gambineri A, Biscotti D, Vicennati V, Gagliardi L, Colitta D, Fiorini S, Cognigni GE, Filicori M, Morselli-Labate AM: Effect of long-term treatment with metformin added to hypocaloric diet on body composition, fat distribution and androgen and insulin levels in abdominally obese women with and without the polycystic ovary syndrome. J Clin Endocrinol Metab 85: 2767-74, 2000.

21- Moran LJ, Noakes M, Clifton PM, Wittert GA, Williams G, Norman RJ: Short-term meal replacements followed by dietary macronutrient restriction enhance weight loss in polycystic ovary syndrome. Am J Clin Nutr 84: 77-87, 2006.

22- Hamilton- Fairley D, Kiddy D, Anyaoku V, Koistinen R, Seppala M, Franks S: Response of sex hormone binding globulin and insulin- like growth factor binding protein-1 to an oral glucose tolerance test in obese women with polycystic ovary syndrome before and after calorie restriction. Clin Endocrinol (Oxf) 39: 363-67, 1993.

23- Poretsky L, Cataldo NA, Rosenwaks Z, Giudice LC: The insulin- related ovarian regulatory system in health and disease. Endocr Rev 20: 535-82, 1999.

24- Kiddy DS, Hamilton-Fairley D, Bush A, Short F, Anyaoku V, Reed MJ, Franks S: Improvement in endocrine and ovarian function during dietary treatment of obese women with polycystic ovary syndrome. Clin Endocrinol (Oxf) 36: 105-11, 1992. 
25- Pasquali R, Antenucci D, Casimirri F, Venturoli S, Paradisi R, Fabbri R, Balestra V, Melchionda N, Barbara L: Clinical and hormonal characteristics of obese amenorrheic hyperandrogenic women before and after weight loss. J Clin Endocrinol Metab 68: 173-9, 1989. 26-Fulghesu AM, Cucinelli F, Pavone V, Murgia F, Guido M, Caruso A, Mancuso S, Lanzone A: Changes in luteinizing hormone and insulin secretion in polycystic ovarian syndrome. Hum Reprod 14: 611-7, 1999.

27- Guzick DS, Wing R, Smith D, Berga SL, Winters SJ: Endocrine consequences of weight loss in obese hyperandrogenic, anovulatory women. Fertil Steril 61: 598-604, 1994.

28- Ebbeling CB, Leidig MM, Sinclair KB, Hangen JP, Ludwig DS: A reduced- glycemic load diet in the treatment of adolescent obesity. Arch Pediatr Adolesc Med 157: 773-79, 2003.

29- Kirwan JP, Barkoukis H, Brooks LM, Marchetti CM, Stetzer BP, Gonzalez F: Exercise training and dietary glycemic load may have synergistic effects on insulin resistance in older obese adults. Ann Nutr Metab 55: 326-33, 2009.

30-Hutchison SK, Stepto NK, Harrison CL, Moran LJ, Strauss BJ, Teede HJ: Effects of exercise on insulin resistance and body composition in overweight and obese women with and without polycystic ovary syndrome. J Clin Endocrinol Metab 96: E48-56, 2011.

31- Yudkin JS, Stehouwer CH, Emeis JJ, Coppack SW: C-reactive protein in healthy subjects: associations with obesity, insulin resistance, and endothelial dysfunction: a potential role for cytokines originating from adipose tissue? Arterioscler Thromb Vasc Biol 19: 927-8, 1999.

32- Florakis D, Diamanti - Kandarakis E, Katsikis I, Nassis GP, Karkanaki A, Georgopoulos N, Panidis D: Effect of hypocaloric diet plus sibutramine treatment on hormonal and metabolic features in overweight and obese women with polycystic ovary syndrome: a randomized, $24-$ week study. Int J Obes (Lond) 32: 692-9, 2008. 
33- Bravata DM, Sanders L, Huang J, Krumholz HM, Olkin I, Gardner CD, Bravata DM: Efficacy and safety of low- carbohydrate diets: a systematic review. JAMA 289: 1837-50, 2003. 34- Noakes M, Keogh JB, Foster PR, Clifton PM: Effect of an energy- restricted, high- protein, low-fat diet relative to a conventional high- carbohydrate, low-fat diet on weight loss, body composition, nutritional status, and markers of cardiovascular health in obese women. Am J Clin Nutr 81: 1298-306, 2005.

35- Liu S, Manson JE, Stampfer MJ, Holmes MD, Hu FB, Hankinson SE, Willett WC: Dietary glycemic load assessed by food- frequency questionnaire in relation to plasma high- density lipoprotein cholesterol and fasting plasma triacylglycerols in postmenopausal women. Am J Clin Nutr 73: 560-6, 2001.

36- Liu S, Manson JE, Buring JE, Stampfer MJ, Willett WC, Ridker PM: Relation between a diet with a high glycemic load and plasma concentrations of high- sensitivity C- reactive protein in middle- aged women. Am J Clin Nutr 75: 492-8, 2002.

37-Esposito K, Pontillo A, Di Palo C, Giugliano G, Masella M, Marfella R, Giugliano D: Effect of weight loss and lifestyle changes on vascular inflammatory markers in obese women: a randomized trial. JAMA 289: 1799-1804, 2003.

38- Moran LJ, Noakes M, Clifton PM, Wittert GA, Belobrajdic DP, Norman RJ: C-reactive protein before and after weight loss in overweight women with and without polycystic ovary syndrome. J Clin Endocrinol Metab 92: 2944-51, 2007.

39- Dvorakova-Lorenzova A, Suchanek P, Havel PJ, Stavek P, Karasova L, Valenta Z, Tintera J, Poledne R: The decrease in C-reactive protein concentration after diet and physical activity induced weight reduction is associated with changes in plasma lipids, but not interleukin-6 or adiponectin. Metabolism 55: 359-65, 2006. 
40- Kasim- Karakas S.E, Almario RU, Gregory L, Wong R, Todd H, Lasley BL: Metabolic and endocrine effects of a polyunsaturated fatty acid- rich diet in polycystic ovary syndrome. J Clin Endocrinol Metab 89: 615-20, 2004.

41- Bobbert T, Rochlitz H, Wegewitz U, Akpulat S, Mai K, Weickert MO, Mohlig M, Pfeiffer AF, Spranger J: Changes of adiponectin oligomer composition by moderate weight reduction. Diabetes 54: 2712-19, 2005. 
Table-1- Baseline measurements of two study groups

\begin{tabular}{lcc}
\hline & CHCD & MHCD \\
& $(\mathrm{n}=26)$ & $(\mathrm{n}=23)$ \\
\hline Age (years) & $28.5 \pm 5.2$ & $30.5 \pm 6.4$ \\
Weight $(\mathrm{kg})$ & $78.9 \pm 12.4$ & $83.0 \pm 11.7$ \\
BMI (kg/m $\left.{ }^{2}\right)$ & $31.1 \pm 4.6$ & $31.9 \pm 4.0$ \\
Waist circumference (cm) & $102.3 \pm 9.9$ & $108.2 \pm 8.1$ \\
Hip circumference (cm) & $112.1 \pm 11.3$ & $120.2 \pm 17.1 *$ \\
Suprailiac skinfold (mm) & $40.0 \pm 9.4$ & $44.1 \pm 15.7$ \\
Total fat mass $(\%)$ & $39.9 \pm 7.1$ & $40.4 \pm 7.5$ \\
Total lean body mass $(\%)$ & $59.9 \pm 7.2$ & $58.8 \pm 7.4$ \\
\hline
\end{tabular}

Values are means \pm SD.

$\mathrm{CHCD}=$ Conventional Hypocaloric Diet, $\mathrm{MHCD}=$ Modified Hypocaloric Diet. There were no baseline differences between the subjects in the 2 groups except for hip circumference $(p<0.05)$. 
Table 2- Calculated dietary composition of MHCD and CHCD from $4 \times 3$ - days food record in comparison to baseline dietary intakes

\begin{tabular}{|c|c|c|c|c|c|c|c|c|}
\hline & \multicolumn{4}{|c|}{ CHCD $(n=26)$} & \multicolumn{4}{|c|}{$\operatorname{MHCD}(n=23)$} \\
\hline & $\begin{array}{c}\text { Prescribed } \\
\text { diet }\end{array}$ & Baseline & Recalls & pvalue & $\begin{array}{c}\text { Prescribed } \\
\text { diet }\end{array}$ & Baseline & Recalls & pvalue \\
\hline $\operatorname{Energy}(\mathrm{kJ} / \mathrm{d})$ & $5021-7113$ & $9117 \pm 515$ & $6832 \pm 414$ & & $5021-7113$ & $9472 \pm 473$ & $6590 \pm 280$ & \\
\hline (Kcal/d) & $1200-1700$ & $2179 \pm 123$ & $1633 \pm 99$ & 0.000 & $1200-1700$ & $2264 \pm 113$ & $1575 \pm 67$ & 0.000 \\
\hline Carbohydrate(\% E) & 55 & $57.1 \pm 1.7$ & $55.2 \pm 4.9$ & 0.125 & 40 & $54.7 \pm 1.5$ & $41.4 \pm 2.7$ & 0.000 \\
\hline Fat $(\% \mathrm{E})$ & 30 & $31.7 \pm 2.0$ & $30.2 \pm 4.6$ & 0.362 & 30 & $33.7 \pm 1.6$ & $29.1 \pm 2.4$ & 0.016 \\
\hline Protein $(\% \mathrm{E})$ & 15 & $11.2 \pm 0.5$ & $14.6 \pm 2.3$ & 0.000 & 30 & $11.6 \pm 2.5$ & $29.5 \pm 2.5$ & 0.000 \\
\hline
\end{tabular}

Values are means \pm SE.

$\mathrm{CHCD}=$ Conventional Hypocaloric Diet, $\mathrm{MHCD}=$ Modified Hypocaloric Diet; \% E, percentage of total daily energy intake.

Pvalue for changes; Recalls- baseline. 
Table 3- Serum concentrations of lipids and reproductive hormones at baseline and after intervention

\begin{tabular}{|c|c|c|c|c|c|c|}
\hline & \multicolumn{3}{|c|}{$\mathrm{CHCD}(\mathrm{n}=26)$} & \multicolumn{3}{|c|}{$\operatorname{MHCD}(n=23)$} \\
\hline & Baseline & week12 & change & Baseline & week12 & change \\
\hline $\mathrm{T}(\mathrm{ng} / \mathrm{ml})$ & $1.5 \pm 0.2$ & $1.1 \pm 0.1^{*}$ & $-0.4 \pm 0.04$ & $1.8 \pm 0.3$ & $1.3 \pm 0.2 \dagger$ & $-0.5 \pm 0.2$ \\
\hline A4 (ng/dl) & $1.9 \pm 0.1$ & $1.8 \pm 0.2$ & $-0.1 \pm 0.1$ & $1.9 \pm 0.1$ & $1.8 \pm 0.1$ & $-0.1 \pm 0.1$ \\
\hline DHEAS (ng/ml) & $330.1 \pm 30.6$ & $298.1 \pm 28.9 \dagger$ & $-32.0 \pm 8.9$ & $314.9 \pm 31.9$ & $272.8 \pm 28.9 \dagger$ & $-42.1 \pm 16.1$ \\
\hline SHBG (nmol/L) & $26.9 \pm 3.8$ & $37.6 \pm 4.6 \ddagger$ & $10.6 \pm 4.1$ & $22.7 \pm 4.0$ & $31.4 \pm 4.4 \dagger$ & $8.8 \pm 2.8$ \\
\hline FSH (IU/L) & $5.2 \pm 0.6$ & $4.5 \pm 0.5$ & $-0.7 \pm 0.5$ & $5.6 \pm 0.8$ & $4.9 \pm 0.6$ & $-0.7 \pm 0.9$ \\
\hline LH (IU/L) & $2.9 \pm 0.4$ & $2.3 \pm 0.4$ & $-0.6 \pm 0.4$ & $2.2 \pm 0.2$ & $2.6 \pm 0.5$ & $0.4 \pm 0.6$ \\
\hline FAI & $6.6 \pm 0.7$ & $4.2 \pm 0.5^{*}$ & $-2.4 \pm 0.5$ & $7.0 \pm 0.9$ & $5.1 \pm 0.8 \dagger$ & $-1.9 \pm 0.7$ \\
\hline Triglyceride (mg/dl) & $132.9 \pm 7.8$ & $124.4 \pm 7.5$ & $-8.6 \pm 5.4$ & $128.7 \pm 6.5$ & $122.4 \pm 5.7$ & $-6.3 \pm 6.1$ \\
\hline Cholesterol (mg/dl) & $197.1 \pm 5.3$ & $191.6 \pm 4.8$ & $-5.5 \pm 3.2$ & $199.9 \pm 5.8$ & $191.5 \pm 5.5$ & $-8.4 \pm 4.1$ \\
\hline HDL-C (mg/dl) & $62.4 \pm 1.6$ & $61.3 \pm 1.2$ & $-1.1 \pm 0.8$ & $59.6 \pm 1.8$ & $59.2 \pm 1.6$ & $-0.4 \pm 0.7$ \\
\hline LDL-C (mg/dl) & $158.5 \pm 6.9$ & $120.0 \pm 6.6^{*}$ & $-38.5 \pm 4.9$ & $158.1 \pm 5.5$ & $116.7 \pm 4.3^{*}$ & $-41.3 \pm 4.3$ \\
\hline
\end{tabular}

Data are mean $\pm \mathrm{SE}$. $\mathrm{CHCD}=$ conventional hypocaloric diet; $\mathrm{MHCD}=$ modified hypocaloric diet; $\mathrm{T}=$ testosterone; $\mathrm{A} 4=$ androstenedione; $\mathrm{DHEAS}=$ dehydroepiandrosterone; $\mathrm{SHBG}=$ sex hormone binding globulin; $\mathrm{FSH}=$ follicle stimulating hormone; $\mathrm{LH}=$ luteinizing hormone; $\mathrm{FAI}=$ free androgen index; HDL-C $=$ High density lipoprotein cholesterol; LDL-C $=$ low density lipoprotein cholesterol

${ }^{*} p<0.001, \dagger p<0.01, \ddagger p<0.05$ 
Table 4- Serum concentrations of insulin and inflammatory factors at baseline and after intervention

$$
\text { CHCD }(n=26) \quad \operatorname{MHCD}(n=23)
$$

\begin{tabular}{lcccccc}
\cline { 2 - 6 } & Baseline & week12 & Change & Baseline & week12 & change \\
\hline Insulin (mU/ml) & $12.2 \pm 6.0$ & $11.2 \pm 5.3$ & $-1.0 \pm 0.7$ & $12.1 \pm 3.3$ & $8.4 \pm 3.7^{*}$ & $-3.6 \pm 0.7 \dagger$ \\
HOMA-IR & $2.9 \pm 1.6$ & $2.7 \pm 1.5$ & $-0.3 \pm 0.2$ & $2.9 \pm 1.4$ & $2.0 \pm 0.9^{*}$ & $-0.8 \pm 0.2 \dagger$ \\
$\mathrm{TNF} \alpha(\mathrm{pg} / \mathrm{ml})$ & $6.3 \pm 0.9$ & $3.8 \pm 0.8 \dagger$ & $-2.5 \pm 1.1$ & $5.4 \pm 0.9$ & $3.3 \pm 0.6 \dagger$ & $-2.1 \pm 0.8$ \\
$\mathrm{IL}-6(\mathrm{pg} / \mathrm{ml})$ & $1.1 \pm 0.06$ & $1.0 \pm 0.06$ & $-0.1 \pm 0.07$ & $1.3 \pm 0.1$ & $1.3 \pm 0.3$ & $0 \pm 0.2$ \\
$\mathrm{hsCRP}(\mathrm{mg} / \mathrm{L})$ & $2.7 \pm 0.6$ & $2.6 \pm 0.6$ & $-0.1 \pm 0.2$ & $3.6 \pm 0.8$ & $2.7 \pm 0.6 \dagger$ & $-0.9 \pm 0.4$ \\
Adiponectin(pg/ml) & $59.6 \pm 4.4$ & $67.7 \pm 4.6 \dagger$ & $8.1 \pm 3.9$ & $59.9 \pm 2.8$ & $68.6 \pm 3.3^{*}$ & $8.7 \pm 2.0$ \\
\end{tabular}

Data are mean $\pm \mathrm{SE} . \mathrm{CHCD}=$ conventional hypocaloric diet; $\mathrm{MHCD}=$ modified hypocaloric diet; ${ }^{*} p<0.001, \dagger p<0.05$

The difference between baseline and week 12 are shown on week 12 in each group. The difference between the changes of two groups are shown on the change of MHCD 


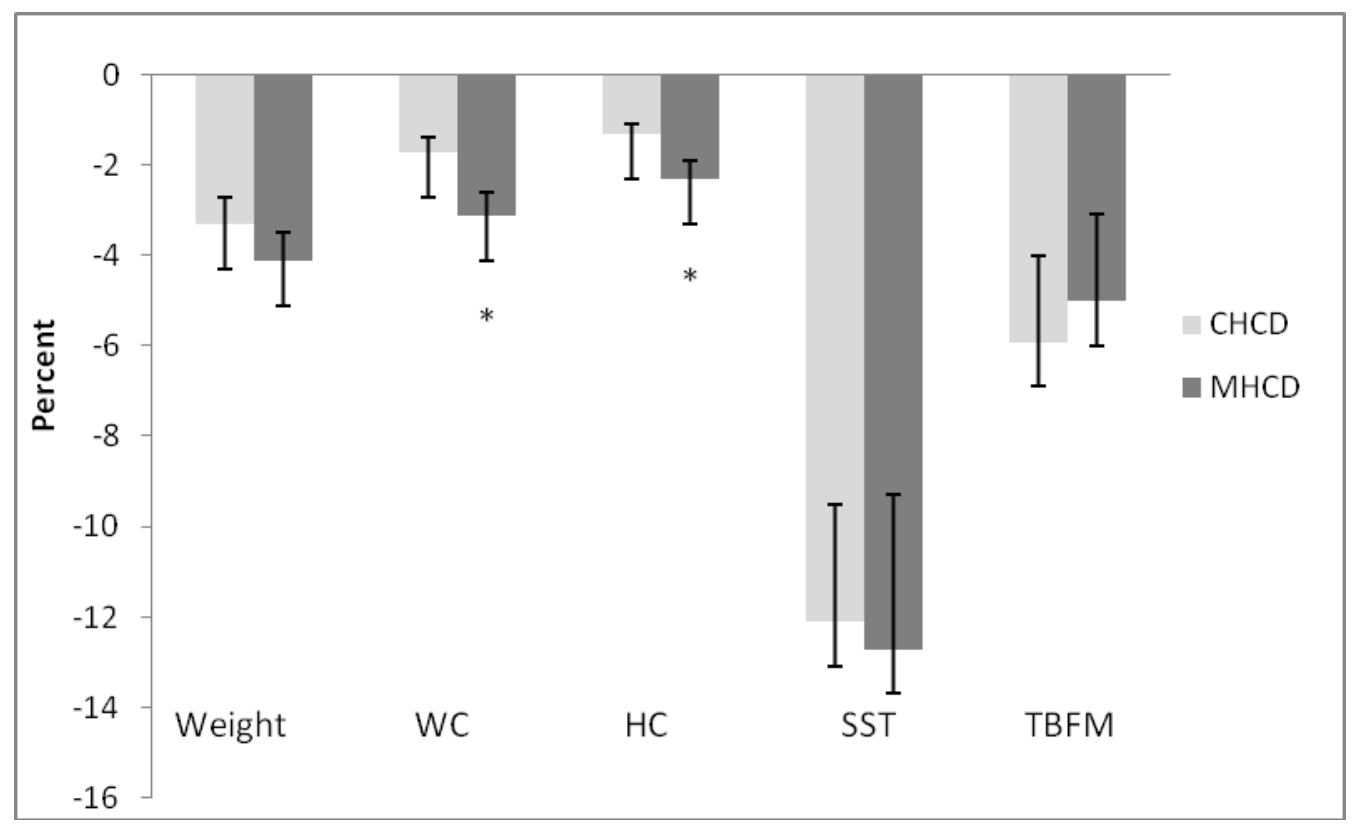

Fig.1 


\section{Legend of figure1}

Fig.1- Anthropometric changes (\%) during 12 weeks use of hypocaloric CHCD and MHCD diet.

$\mathrm{CHCD}=$ conventional hypocaloric diet; $\mathrm{MHCD}=$ modified hypocaloric diet; $\mathrm{WC}=$ waist circumference, $\mathrm{HC}=$ hip circumference, $\mathrm{SST}=$ suprailiac skinfold thickness, $\mathrm{TBFM}=$ total body fat mass.

All the anthropometric changes were significant for both groups; CHCD and MHCD $(p<0.05)$, except for TBFM.

$* p<0.05$ in comparison to CHCD. 